\title{
Pacific
}

Journal of

Mathematics

\section{ON SURGERY CURVES FOR GENUS-ONE SLICE KNOTS}

PATRICK M. GILMER AND ChARLES Livingston 


\title{
ON SURGERY CURVES FOR GENUS-ONE SLICE KNOTS
}

\author{
PATRICK M. GILMER AND CHARLES LiVINGSTON
}

\begin{abstract}
If a knot $K$ bounds a genus-one Seifert surface $F \subset S^{3}$ and $F$ contains an essential simple closed curve $\alpha$ that has induced framing 0 and is smoothly slice, then $K$ is smoothly slice. Conjecturally, the converse holds. It is known that if $K$ is slice and the determinant of $K$ is not 1 , then there are strong constraints on the algebraic concordance class of such $\alpha$, and it was thought that these constraints might imply that $\alpha$ is at least algebraically slice. We present a counterexample; in the process we answer negatively a question of Cooper and relate the result to a problem of Kauffman. Results of this paper depend on the interplay between the Casson-Gordon invariants of $K$ and algebraic invariants of $\alpha$.
\end{abstract}

\section{Introduction}

For $n>1$, if a smooth knotted $S^{2 n-1}$ in $S^{2 n+1}$ bounds an embedded disk in $B^{2 n+2}$, such a smooth slicing disk can be constructed from a $2 n$-manifold bounded by $K$ in $S^{2 n+1}$ by ambient surgery. Whether the same is true for knots in $S^{3}$ has remained an open question for 40 years, though counterexamples exist in the topological category [Freedman and Quinn 1990].

One well-known and simply stated conjecture [Kirby 1978, Problem 1.38] is a special case: the untwisted Whitehead double of a knot $J \subset S^{3}$ is smoothly slice if and only if $J$ is smoothly slice. More generally, if $K$ is a knot in $S^{3}$ that bounds a genus-one Seifert surface $F$ and is algebraically slice, then up to isotopy and orientation change, there are exactly two essential simple closed curves on $F, J_{1}$ and $J_{2}$, with self-linking 0 with respect to the Seifert form of $F$. In this situation, we will call $J_{1}$ and $J_{2}$ surgery curves for $F$. Conjecturally, if $K$ is smoothly slice, then one of $J_{1}$ or $J_{2}$ is necessarily smoothly slice (see [Kauffman 1987, Strong conjecture, page 226] for instance).

Shortly after Casson and Gordon [1986] developed obstructions to slicing algebraically slice knots, it was noticed that Casson-Gordon invariants could be

Gilmer was partially supported by NSF-DMS-0905736. Livingston was partially supported by NSF-DMS-1007196.

MSC2010: 57M25.

Keywords: knot, slice knot, genus 1. 
expressed in terms of signature invariants of curves on Seifert surfaces [Gilmer 1983; Litherland 1984]. Moreover, Casson-Gordon invariants could be interpreted in this way as obstructions to slicing $K$ by slicing a surgery curve on a genus-one Seifert surface for $K$. Casson-Gordon invariants actually obstruct topological locally flat slice disks.

A genus-one knot $K$ is algebraically slice if and only if it has an Alexander polynomial of the form

$$
\begin{aligned}
\Delta_{K}(t) & =(m t-(m+1))((m+1) t-m) \\
& =m(m+1) t^{2}-\left(m^{2}+(m+1)^{2}\right) t+m(m+1)
\end{aligned}
$$

for some $m \geq 0$. Observe that if $\Delta_{K}$ has the form above, then the nonnegative integer $m$ is determined. For a genus-one algebraically slice knot $K$, let $m(K)$ denote this number; note that the determinant of $K$ is $(2 m(K)+1)^{2}$.

We let $\sigma_{K}(t)$ denote the Levine-Tristram signature function of $K$ [Levine 1969; Tristram 1969], as defined on the unit interval $[0,1]$ and redefined to be the average of the one-sided limits at the jumps. Casson-Gordon theory implies that if a genusone knot $K$ is slice and $m(K) \neq 0$, then the signature function of one of the surgery curves satisfies strong constraints. To state these, we make the following definition.

Definition 1. A knot $J$ satisfies the $(m, p)$-signature conditions for integers $m>0$ and $p$ relatively prime to $m$ and $m+1$ if

$$
\sum_{i=0}^{r-1} \sigma_{J}\left(c a^{i} / p\right)=0
$$

for all $c \in \mathbb{Z}_{p}{ }^{*}$, and $a=(m+1) / m$ mod $p$,where $r$ is the order of $a$ modulo $p$.

To get a feeling for this summation, consider the case of $m(K)=1$ and $p=73$. In $\mathbb{Z}_{73}$, the number 2 generates the multiplicative subgroup $\{1,2,4,8,16,32,64,55,37\}$. This subgroup has 8 cosets in the group of units $\left(\mathbb{Z}_{73}\right)^{*}$. For instance, the coset containing $c=5$ is $\{5,7,10,14,20,28,39,40,56\}$. Thus the following arises as one of the sums in the $(1,73)$-signature condition:

$\sigma_{J}\left(\frac{5}{73}\right)+\sigma_{J}\left(\frac{7}{73}\right)+\sigma_{J}\left(\frac{10}{73}\right)+\sigma_{J}\left(\frac{14}{73}\right)+\sigma_{J}\left(\frac{20}{73}\right)+\sigma_{J}\left(\frac{28}{73}\right)+\sigma_{J}\left(\frac{39}{73}\right)+\sigma_{J}\left(\frac{40}{73}\right)+\sigma_{J}\left(\frac{56}{73}\right)$.

Notice that the cosets appear to be fairly randomly distributed in the unit interval. Nonetheless, as we show, the vanishing of all such sums is not sufficient to imply the vanishing of the signature function itself. Consider the following simple consequence of Theorem 8 below.

Theorem 2. If $K$ is a genus-one smoothly slice knot, then one of the surgery curves $J$ satisfies $(m(K), p)$-signature conditions for an infinite set of primes $p$.

In his thesis, Cooper states a stronger result: 
Theorem 3 [Cooper 1982]. Let $K$ be a genus-one smoothly slice knot, then one of the surgery curves $J$ satisfies the $(m(K), p)$-signature conditions for all $p$ relatively prime to $m$ and $m+1$.

One quick corollary, first observed by Cooper, of either of these theorems is that for a genus one slice knot $K$ with $m(K)>0$, the integral of the signature function of one of the slice curves $J$ is 0 . This follows by summing the signature sums in the theorem over all values of $c$ to get a sum of the form $\sum_{i=1}^{p-1} \sigma_{J}(i / p)=0$ and then noting that for large $p$, this sum approximates the integral. (This integral condition was later seen to follow from the $L^{2}$-signature approach of [Cochran et al. 2003, Theorem(1.4)].)

Clearly, the constraints given by these theorems are quite extensive. One explicit question asked by Cooper is whether the vanishing of the combined sum $\sum_{i=1}^{p-1} \sigma_{J}(i / p)$ for the appropriate infinite sets of $p$ implies the vanishing of the signature function [Cooper 1982, Question (3.16)]. We will show that the answer is no. In fact, the much stronger constraints given in Theorems 2 and 3 are not sufficient to imply the vanishing of the signature function of one of the surgery curves. Here is the algebraic formulation of the question.

Question 4. Let $\sigma$ be an integer-valued step function defined on $[0,1]$ with the property that $\sigma(x)=\sigma(1-x)$ for all $x$. Assume also $\sigma(0)=\sigma(1)=0$, that there are no jumps at points with denominator a prime power, and that $\sigma$ is equal to the average of the one-sided limits at the jumps. Suppose that for all $p>1$ coprime to $m$ and $m+1$, for $G$ the multiplicative subgroup of $\left(\mathbb{Z}_{p}\right)^{*}$ generated by $(m+1) / m$, and for all $n \in \mathbb{Z}_{p}$, we have

$$
\sum_{r \in n G} \sigma(r / p)=0
$$

Then does $\sigma(t)=0$ for all $t$ ?

For each $m>0$, the answer to the above question is emphatically no. Let $K_{(r, s)}$ denote the $(r, s)$-cable of $K$ (that is, $r$ longitudes, and $s$ meridians). Let $-K$ denote the mirror image of $K$.

Theorem 5. Let $K$ be a knot with a nonzero signature function, and $m>0$. The signature function of $K_{(m, 1)} \#-K_{(m+1,1)}$ is nonzero and satisfies the $(m, p)$-signature conditions for all $p$ relatively prime to $m$ and $m+1$.

We have a perhaps nicer family to work with in the case $m=1$. Let $T_{r, s}$ denote the $(r, s)$-torus knot, which is the $(r, s)$-cable of the unknot.

Theorem 6. If $r$ is an odd number and $r \geq 3$, the signature function of $\left(T_{2, r}\right)_{(2,-r)}$ is nonzero and satisfies the $(1, p)$-signature conditions for odd $p$. 


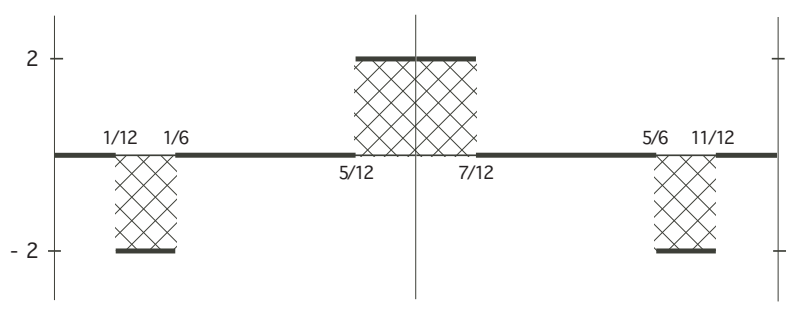

Figure 1. Signature function of $\left(T_{2,3}\right)_{(2,-3)}$ satisfying the $(1, p)$-signature conditions for odd $p$.

Although Casson-Gordon theory gives a somewhat weaker version of Cooper's theorem, it provides access to the more powerful Witt class analogs of Theorem 2, which carry more information than is given by signatures. Also, Casson-Gordon theory obstructs topological sliceness, whereas Cooper worked in the smooth category. We now describe these Witt class invariants.

If $K$ is a knot, let $V_{t}=(1-t) V+\left(1-t^{-1}\right) V^{t}$, where $V$ is a Seifert matrix of $K$ and $t$ is an indeterminate. For $p$ a prime power and $j / p \in \mathbb{Z}[1 / p] / \mathbb{Z}$, let $w_{K}(j / p)$ denote the element represented by $V_{e^{2 \pi i j / p}}$ in $W\left(\mathbb{Q}\left(\zeta_{p}\right)\right) \otimes \mathbb{Z}_{(2)}$. Here, $W\left(\mathbb{Q}\left(\zeta_{p}\right)\right)$ denotes the Witt group of hermitian forms over the field $\mathbb{Q}\left(\zeta_{p}\right)$ and $\mathbb{Z}_{(2)}$ denotes $\mathbb{Z}$ localized at 2 . An elementary proof shows that this defines a homomorphism on the concordance group.

Definition 7. We say a knot $J$ satisfies the $(m, p)$-Witt conditions for integers $m>0$ and $p$ relatively prime to $m$ and $m+1$ if

$$
\sum_{i=0}^{r-1} w_{J}[(c+a i) / p]=0 \in W\left(\mathbb{Q}\left(\zeta_{p}\right)\right) \otimes \mathbb{Z}_{(2)}
$$

for all $c \in \mathbb{Z}_{p}{ }^{*}, a=(m+1) / m \bmod p$, and $r$ the order of $a$ modulo $p$.

If a knot $J$ satisfies the $(m, p)$-Witt conditions, it satisfies the $(m, p)$-signature conditions as well. But the Witt conditions are stronger. For instance, one can define a discriminant invariant on $W\left(\mathbb{Q}\left(\zeta_{p}\right)\right) \otimes \mathbb{Z}_{(2)}$, which is discussed in [Gilmer and Livingston 1992b].

Theorem 8. Let $K$ be a genus one topologically slice knot. There is some finite set of bad primes $P$ such that one of the surgery curves $J$ satisfies the $(m(K), p)$-Witt conditions for all $p$ in the set $\left\{r^{n} \mid n \in \mathbb{Z}_{+}, r\right.$ is prime, $r \notin P, r^{n}$ divides $(m+1)^{q}-(m)^{q}$ for some prime power $\left.q\right\}$.

Consider $\mathrm{Wh}(J, n)$, the $n$-twisted Whitehead double of $J$. It is well-known that this knot is algebraically slice if and only if $n=m(m+1)$. Moreover $m(\operatorname{Wh}(J, m(m+1)))=m$. It is also known that the two surgery curves for 
$\mathrm{Wh}(J, m(m+1))$ both have the isotopy type of $J \# T_{(m, m+1)}$. One can see this using the techniques discussed in [Kauffman 1987, pages 214-223]. Using this fact, for these knots one can sometimes remove the exceptions created by the unknown set of bad primes.

Theorem 9. Let $m>0$. If $\mathrm{Wh}(J, m(m+1))$ is topologically slice, then $J \# T_{(m, m+1)}$ satisfies the $(m, p)$-Witt conditions for all $p$ in the set

$\left\{p \mid p\right.$ is prime, $\operatorname{gcd}\left(p^{2},(m+1)^{q}-(m)^{q}\right)=p$ for some odd prime power $\left.q\right\}$.

Our examples of knots satisfying $(m, p)$-signature conditions also satisfy Witt conditions.

Theorem 10. For any knot $K$ and $m>0, K_{(m, 1)} \#-K_{(m+1,1)}$ satisfies the $(m, p)$ Witt conditions for all $p$ relatively prime to $m$ and $m+1$. For any odd integer $n$, $\left(T_{2, n}\right)_{(2,-n)}$ satisfies the $(1, p)$-Witt conditions for all odd $p$.

In the next theorems, we focus on some particularly nice examples.

Theorem 11. Let $J=\left(T_{2,3}\right)_{(2,-3)}$, the $(2,-3)$-cable of trefoil knot $T_{2,3}$. Let $K=\mathrm{Wh}(J, 2)$.

(1) $K$ is a genus one algebraically slice knot with both surgery curves having the same knot type, $J$.

(2) $J$ satisfies the $(1, p)$-Witt conditions for all odd $p$. In particular $J$ satisfies the $(1, p)$-signature conditions for all odd $p$. Another consequence is that the constraints of Theorems 3,8 , and 9 on $K$ are satisfied.

(3) The signature function of $J$ is nonzero.

(4) $\Delta_{J}(t)=\left(t^{-1}-1+t\right)\left(t^{-2}-1+t^{2}\right)$ does not satisfy the Fox-Milnor condition; that is, $\Delta_{J}(t)$ cannot be written as $f(t) f\left(t^{-1}\right)$ for $f(t) \in \mathbb{Z}\left[t, t^{-1}\right]$.

(5) $\operatorname{Arf} J \neq 0$.

We do not know whether $\operatorname{Wh}\left(\left(T_{2,3}\right)_{(2,-3)}, 2\right)$ is topologically locally flat slice or smoothly slice. A conjecture made by Kauffman [1987, Weak Conjecture, page 226; Kirby 1978, Problem 1.52] implies that $\mathrm{Wh}\left(\left(T_{2,3}\right)_{(2,-3)}, 2\right)$ is not smoothly slice since $\operatorname{Arf}\left(\left(T_{2,3}\right)_{(2,-3)}\right) \neq 0$. Thus examples such as this one offer a route to possible counterexamples to this conjecture.

By modifying the example slightly (without changing the relevant signature function, Alexander polynomial, Arf invariant, or even Witt class invariant), using results of Hedden [2007; 2009] on the Ozsváth-Szabó invariant of cables and Whitehead doubles, obstructing sliceness becomes possible. This is described in the first part of the following theorem. We also give a second example of a knot with similar properties. 
Theorem 12. Let $J^{\prime}=\left(T_{2,3} \# \mathrm{Wh}\left(T_{2,3}, 0\right)\right)_{(2,-3)}$. Then $K^{\prime}=\mathrm{Wh}\left(J^{\prime}, 2\right)$ is not smoothly slice. Moreover the conclusions of Theorem 11 hold when $K$ is replaced by $K^{\prime}$ and $J$ is replaced by $J^{\prime}$.

Let $J^{\prime \prime}=\left(T_{2,3}\right)_{(2,-3)} \#\left(T_{2,3}\right)_{(2,-3)}$. Then $K^{\prime \prime}=\mathrm{Wh}\left(J^{\prime \prime}, 2\right)$ is not smoothly slice. Moreover conclusions (1), (2), and (3) of Theorem 11 hold when $K$ is replaced by $K^{\prime \prime}$ and $J$ is replaced by $J^{\prime \prime}$.

In Section 2, we outline the proofs of Theorems 5, 6 and 10. Section 3 presents the proof of Theorem 12 using tools from Heegaard-Floer theory. In Section 4 and Appendices B and C, we review Casson-Gordon theory and prove Theorems 8 and 9. Similar arguments have appeared, but some depend on Theorem 1 of [Gilmer 1993], whose proof contains a gap (shared with [Gilmer 1983, Theorem (0.1)]). We show how to modify this proof to obtain the results stated above. In Section 5, we give some restrictions on signature functions which satisfy the $m$-signature averaging conditions.

\section{Proofs of Theorems 5, 6 and 10}

Let $S$ be a finite set in $\mathbb{R} / \mathbb{Z}$. For any function $f(t)$ on $\mathbb{R} / \mathbb{Z}$ taking values in an abelian group, define $\mu_{S}(f(t))=\sum_{s \in S} f(s)$. We let $\phi_{k}: \mathbb{R} / \mathbb{Z} \rightarrow \mathbb{R} / \mathbb{Z}$ denote multiplication by the integer $k$. Observe that if $\phi_{k}$ is injective on $S$, then $\mu_{\phi_{k}(S)}(f(t))=\mu_{S}(f(k t))$. In particular, we have the following.

Lemma 13. If $S \subset \mathbb{R} / \mathbb{Z}$ is a finite set on which $\phi_{m}$ and $\phi_{n}$ are both injective and $\phi_{m}(S)=\phi_{n}(S)$, then for all $f, \mu_{S}(f(m t)-f(n t))=0$.

In the current case of interest, we have an integer $m$, an integer $p$ relatively prime to $m(m+1)$, and an integer $c$ representing an element in $\mathbb{Z}_{p}^{*}$. We let $a=(m+1) / m$ $\bmod p$ and $S=\left\{c a^{i} / p\right\} \subset \mathbb{Q} / \mathbb{Z}$. Notice that $m a^{i}=(m+1) a^{i-1}$. Thus, in this setting $\phi_{m}(S)=\phi_{m+1}(S)$.

Corollary 14. With the notation of the previous paragraph, for all $f$,

$$
\mu_{S}(f((m+1) t)-f(m t))=0 .
$$

An immediate application is the case that $f$ is the signature function of a knot $J$, in which case $f(m t)$ is the signature function of the knot $J_{m, \pm 1}$.

In the proof of Lemma 13, it is not required that $f$ be defined on all of $\mathbb{R} / \mathbb{Z}$, but only on the sets $S, \phi_{m}(S)$ and $\phi_{n}(S)$. For instance, for a knot $J$ and prime power $p$, there is the function $w_{J}:\{j / p\} \rightarrow W\left(\mathbb{Q}\left(\zeta_{p}\right)\right) \otimes \mathbb{Z}_{(2)}$ defined by

$$
w_{J}(j / p)=\left(1-\zeta_{p}^{j}\right) V+\left(1-\zeta_{p}^{-j}\right) V^{t},
$$

where $\zeta_{p}=e^{2 \pi i / p}$. 
The only missing ingredient in the proofs of Theorems 5, 6, and 10 is the following theorem.

Theorem 15. If $S$ is a satellite of $C$ with orbit $P$ and winding number $n$, then

$$
w_{\mathbb{S}}(j / p)=w_{P}(j / p)+w_{C}(n j / p) .
$$

This result is very close to a result of Litherland [1984, Theorem 1], which states that if $V_{t}(K)=(1-t) V+\left(1-t^{-1}\right) V^{t}$, where $V$ is the Seifert form of $K$, then $V_{t}(\mathbb{S})$ is Witt equivalent to the form $V_{t^{n}}(C) \oplus V_{t}(P)$ in the Witt group $\mathrm{Wh}(\mathbb{Q}(t))$ of the function field. One would like to argue at this point that the substitution of $\zeta_{p}$ for $t$ defines a map $W(\mathbb{Q}(t)) \rightarrow W\left(\mathbb{Q}\left(\zeta_{p}\right)\right)$, and Theorem 15 results. Unfortunately, this procedure does not lead to a well-defined map $W(\mathbb{Q}(t)) \rightarrow W\left(\mathbb{Q}\left(\zeta_{p}\right)\right)$, as a class in $W(\mathbb{Q}(t))$ may be represented by a matrix whose entries have poles at $\zeta_{p}$. We leave it to Appendix A to show how this hurdle can be overcome.

\section{Smooth obstructions to slicing}

In [Ozsváth and Szabó 2003], an invariant $\tau$ is defined with the property that if $K$ is smoothly slice, then $\tau(K)=0$. In order to apply this, we need to modify our knot $K$ slightly. Let $K^{\prime}=\mathrm{Wh}\left(\left(T_{2,3} \# \mathrm{Wh}\left(T_{2,3}, 0\right)\right)_{(2,-3)}, 2\right)$. We show $\tau\left(K^{\prime}\right)=1$.

As a first step, it follows from [Ozsváth and Szabó 2003] that $\tau\left(T_{2,3}\right)=1$. Next, Hedden [2007] proved that for any $J, \tau(\mathrm{Wh}(J, t))=1$ for all $t<2 \tau(J)$. Thus, $\tau\left(\mathrm{Wh}\left(T_{2,3}, 0\right)\right)=1$. By additivity, $\tau\left(T_{2,3} \# \mathrm{Wh}\left(T_{2,3}, 0\right)\right)=2$.

According to another theorem of Hedden [2009], if $\tau(J)=$ genus $(J)$, then

$$
\tau\left(J_{(s, s n+1)}\right)=s \tau(J)+\frac{1}{2} s n(s-1)+s-1 .
$$

In the case of interest to us, we have $s=2$ and $n=-2$, so $\tau\left(J_{(2,-3)}\right)=2 \tau(J)-1$. We do have $\tau\left(T_{2,3} \# \mathrm{Wh}\left(T_{2,3}, 0\right)\right)=\operatorname{genus}\left(T_{2,3} \# \mathrm{Wh}\left(T_{2,3}, 0\right)\right)=2$, so

$$
\tau\left(\left(T_{2,3} \# \mathrm{Wh}\left(T_{2,3}, 0\right)\right)_{(2,-3)}\right)=2 \tau\left(T_{2,3} \# \mathrm{Wh}\left(T_{2,3}, 0\right)\right)-1=2(2)-1=3 .
$$

Finally, again by Hedden's computation of $\tau$ of doubled knots,

$$
\tau\left(\mathrm{Wh}\left(\left(T_{2,3} \# \mathrm{Wh}\left(T_{2,3}, 0\right)\right)_{(2,-3)}, t\right)\right)=1
$$

if $t<6$. So in particular, $\tau\left(\mathrm{Wh}\left(\left(T_{2,3} \# \mathrm{Wh}\left(T_{2,3}, 0\right)\right)_{(2,-3)}, 2\right)\right)=1$.

We can also consider $K^{\prime \prime}=\mathrm{Wh}\left(\left(T_{2,3}\right)_{(2,-3)} \#\left(T_{2,3}\right)_{(2,-3)}, 2\right)$. Using the same formula of Hedden's for cables, we have

$$
\tau\left(\left(T_{2,3}\right)_{(2,-3)}\right)=1,
$$

which gives us

$$
\tau\left(\left(T_{2,3}\right)_{(2,-3)} \#\left(T_{2,3}\right)_{(2,-3)}\right)=2 .
$$

Then using Hedden's formula for doubles, $\tau\left(K^{\prime \prime}\right)=1$. 


\section{Casson-Gordon theory}

By a character $\chi$ on $X$, we mean a homomorphism $\chi: H_{1}(X) \rightarrow \mathbb{Q} / \mathbb{Z}$. This is a $d$-character if $\chi: H_{1}(X) \rightarrow(1 / d) \mathbb{Z} / \mathbb{Z} \subset \mathbb{Q} / \mathbb{Z}$. Given a knot $K$ and a prime power $q$, let $S_{q}$ denote the $q$-fold branched cyclic cover of $S^{3}$ along $K$. Given a $d$-character on $S_{q}$, Casson and Gordon [1986] defined an invariant $\tau(K, \chi)$ taking values in $W\left(\mathbb{Q}\left[\zeta_{d}\right](t)\right) \otimes \mathbb{Q}$. Here, $W\left(\mathbb{Q}\left[\zeta_{d}\right](t)\right)$ is the Witt group of Hermitian forms over $\mathbb{Q}\left[\zeta_{d}\right](t)$. If $d$ is odd (as will be the case when $K$ is a genus one algebraically slice knot), then $\tau(K, \chi)$ may be refined to take values in $W\left(\mathbb{Q}\left[\zeta_{d}\right](t)\right) \otimes \mathbb{Z}_{(2)}$ [Gilmer and Livingston 1992a; 1992b]. This refinement is useful as these Witt groups have 2 -torsion. Here is the theorem of Casson and Gordon [1986; 1978] which asserts that certain $\tau(K, \chi)$ vanish when $K$ is slice. (Casson and Gordon proved this theorem for smooth slice disks, and later, based on [Freedman and Quinn 1990], it was seen to hold in the topological locally flat category.)

Theorem 16 [Casson and Gordon 1986]. Let $K$ be a slice knot bounding a slice disk $\Delta \subset B^{4}$. Let $W_{q}$ be the q-fold cyclic branched cover of $B^{4}$ over $\Delta$.

- If $\chi$ is a character on $S_{q}$ of prime power order that extends to $W_{q}$, then $\tau(K, \chi)=0$.

- A character $\chi$ on $S_{q}$ extends to $W_{q}$ if and only if it vanishes on $\kappa(\Delta, q)$, the kernel of $H_{1}\left(S_{q}\right) \rightarrow H_{1}\left(W_{q}\right)$.

- The kernel $\kappa(\Delta, q)$ is a metabolizer for the linking form on $H_{1}\left(S_{q}\right)$ and is invariant under the group of covering transformations.

- The set of characters $\chi$ on $S_{q}$ that extend to $W_{q}$ form a metabolizer $\mathfrak{m}(q, \Delta)$ for the linking form on $H^{1}\left(S_{q}, \mathbb{Q} / \mathbb{Z}\right)$.

If $p$ is a prime and $G$ is an abelian group, let $G_{(p)}$ denote the $p$-primary summand of $G$. Note that the obstruction to sliceness given by Theorem 16 can be reduced to a sequence of obstructions associated to each prime $p: \tau(K, \chi)=0$ for $\chi \in$ $\mathfrak{m}(q, \Delta)_{(p)}$.

Let $F$ be a Seifert surface $K$. Then $F \cup \Delta$ bounds a 3-manifold $R \subset B^{4}$. In [Gilmer 1993, Theorem 1], the first author related $\mathfrak{m}(q, \Delta)$ to the metabolizer $H$ for Seifert form on $H_{1}(F)$ that arises as the kernel of the map induced by inclusion $H_{1}(F) \rightarrow H_{1}(R) /$ Torsion $\left(H_{1}(R)\right)$. However, Stefan Friedl [2004] found a gap in the proof, appearing in the second to last sentence on page 6 of [Gilmer 1993]. We now want to state a corrected version of the theorem.

Theorem 17. Assume the notations and suppositions of Theorem 16, and let $R$ and $H$ be as above. Let $p$ be a prime relatively prime to $\left|\operatorname{Torsion}\left(H_{1}(R)\right)\right|$. Let $\left\{x_{i}^{\prime}\right\}$ be a basis for $H$. Let $\left\{y_{i}^{\prime}\right\}$ be a complementary dual basis in $H_{1}(F)$ to $\left\{x_{i}^{\prime}\right\}$ with respect to the intersection pairing. View $F$ as built from a disk by adding $2 g$ bands, with 
cores representing the $x_{i}^{\prime}$ and $y_{i}^{\prime}$. Let the linking circles to those bands be denoted $x_{i}$ and $y_{i}$. Let $\checkmark$ be the subgroup of $H_{1}\left(S_{q}\right)$ generated by the lifts of the $y_{i}$ to a single component of the inverse image of $S^{3} \backslash F$ in $S_{q}$. Then $\kappa(\Delta, q)_{(p)}=\mathbb{Y}_{(p)}$.

Two independent proofs of Theorem 17 are presented in Appendices B and C. In [Friedl 2004, Theorem 8.6] and [Cochran et al. 2003, page 511], an equivalent result is asserted for almost all primes $p$ (rather than for all primes not dividing |Torsion $\left.\left(H_{1}(R)\right) \mid\right)$.

To each element $z \in H_{1}\left(S_{q}\right)_{(p)}$, there is an associated character

$$
\chi_{z}: H_{1}\left(S_{q}\right)_{(p)} \rightarrow \mathbb{Z}_{p^{k}} \subset \mathbb{Q} / \mathbb{Z}
$$

(for some value of $k$ ), defined by $\chi_{z}(w)=\ell k(w, z) \in \mathbb{Q} / \mathbb{Z}$.

Corollary 18. Assuming the notations and suppositions of Theorems 16 and 17, then $\mathfrak{m}(q, \Delta)_{(p)}=\left\{\chi_{z} \mid z \in \mathbb{Y}_{(p)}\right\}$.

We can now summarize the proof of Theorem 8. Details follow as in [Gilmer 1993].

Proof of Theorem 8. By Theorem 17, one needs to show that the vanishing of the Casson-Gordon invariants for characters $\chi_{z}$ with $z \in \mathbb{Y}_{p}$ implies the surgery curve $J$ satisfies the specified $(m(K), p)$-Witt conditions. There are two steps. First, one considers a new knot $K^{\prime}$, formed from $K$ by tying a knot $-J$ in the band of the Seifert surface representing $J$. This new knot is slice, since it has surgery curve $J \#-J$, which is slice. The manifold $R$ for $K^{\prime}$ is built by adding a two-handle to $F \times[0,1]$, and can be seen to be a solid handlebody, in fact, a solid torus. Thus, Theorem 17 implies that for all the relevant characters, the Casson-Gordon invariants vanish. The proof is completed by proving that the effect of changing $K$ to $K^{\prime}$ on the Casson-Gordon invariants is to add the sum of invariants appearing in the $(m(K), p)$-Witt conditions.

(We take this opportunity to remark that Theorem (3.5) of [Gilmer 1983] remains valid. Although its proof uses Theorem 1.1 of the same paper, ${ }^{1}$ it only does so in the case that $R$ is a handlebody. For similar reasons, the proof of [Naik 1996, Theorem 7] is valid.)

Proof of Theorem 9. If $K$ is an algebraically slice knot of genus one, $m=m(K)$, and $q$ is odd, then $H_{1}\left(S_{q}\right)$ is the direct sum of two cyclic groups of order $(m+1)^{q}-m^{q}$. For each odd prime $p$ such that $\operatorname{gcd}\left(p^{2},(m+1)^{q}-(m)^{q}\right)=p$, the $p$-primary part of $H_{1}\left(S_{q}\right)$ (denoted $H_{1}\left(S_{q}\right)_{(p)}$ ) is a two-dimensional vector space over $\mathbb{Z}_{p}$. An analysis of $H_{1}\left(S_{q}\right)$ (as in the proof of [Gilmer 1993, top of page 16]) shows that the two metabolizers for the Seifert form spanned by the two surgery curves, say $J_{1}$ and $J_{2}$, lead to two distinct metabolizers for the linking form restricted to $H_{1}\left(S_{q}\right)_{(p)}$. In

\footnotetext{
${ }^{1}$ This is the same as [Gilmer 1993, Theorem 1] in the case $q=2$.
} 
fact, these metabolizers are eigenspaces for a generator of the group of covering transformations with the distinct eigenvalues $(m+1) / m$ and $m /(m+1)$. Thus this linking form on $H_{1}\left(S_{q}\right)_{(p)}$ is hyperbolic. It follows that an element in $H_{1}\left(S_{q}\right)_{(p)}$ in the complement of the union of these two metabolizers cannot have self-linking zero. Therefore, the linking form on $H_{1}\left(S_{q}\right)_{(p)}$ has only these two metabolizers.

If $K$ is slice, then $\kappa(\Delta, q)_{(p)}$ must be one of these two metabolizers. Thus by Theorem 16, if $\chi: H_{1}\left(S_{q}\right) \rightarrow(1 / p) \mathbb{Z} / \mathbb{Z}$ vanishes on $\kappa(\Delta, q)_{(p)}$, then $\tau(K, \chi)=0$. By [Gilmer 1993, proof of Theorem 3], for each of these $p$, either $J_{1}$ or $J_{2}$ must satisfy the $(m, p)$-Witt conditions. But for $K=W(J, m(m+1))$, both $J_{1}$ and $J_{2}$ have the isotopy type of $J \# T_{(m, m+1)}$.

\section{The averaging conditions restrict where the jumps can occur}

We consider the family $\mathscr{g}$ of step functions $f$ on $[0,1]$ that vanish at 0 and 1 and have a finite number of jumps, with value at the jumps the average of the one-sided limits. For $f \in \mathscr{g}$, define

$$
\Sigma_{p}(f)=\sum_{i=1}^{p-1} f(i / p) .
$$

Consider, also, the family of symmetric jump functions

$$
\mathscr{S}=\{f \in \mathscr{f} \mid f(x)=f(1-x)\} .
$$

These include the knot signature functions.

We say that $\sigma \in \mathscr{Y}$ satisfies the $m$-signature averaging condition if $\Sigma_{p}(\sigma)=0$ for each $p$ relatively prime to $m$ and $m+1$. The $m$-signature averaging condition is a consequence of the $(m, p)$-signature conditions for all $p$ relatively prime to $m$ and $m+1$.

The Alexander polynomial of the knot $5_{2}$ is $2-3 t+2 t^{2}$ [Cha and Livingston 2011] which has simple roots at $\frac{1}{4}(3 \pm i \sqrt{7})$. These roots lie on the unit circle and have argument $\pm 2 \pi a$, where $a=\frac{1}{2 \pi i} \log \left(\frac{1}{4}(3+i \sqrt{7})\right) \approx 0.115$.

Proposition 19. The number a is irrational. The signature function of $5_{2} \#-\left(5_{2}\right)_{2,1}$ has jumps in the interval $\left[0, \frac{1}{2}\right]$ at $a / 2, a$, and $(1-a) / 2$, and this signature function satisfies the $(1, p)$-signature conditions for all odd $p$.

Proof. If $a$ were rational, $2-3 t+2 t^{2}$ would have to be a factor of some cyclotomic polynomial; but these are monic. The signature function of $5_{2}$ viewed as a function on [0,1] has jumps at $a$ and $1-a$. Using [Litherland 1984] or [Livingston and Melvin 1985], the signature function of the knot $\left(5_{2}\right) \#-\left(5_{2}\right)_{2,1}$ jumps at exactly $a / 2, a,(1-a) / 2,(1+a) / 2,1-a$, and $1-a / 2$. By Theorem $5,\left(5_{2}\right) \#-\left(5_{2}\right)_{2,1}$ satisfies the $(1, p)$-signature conditions for all odd $p$. 
This example contradicts a claim that we once (see the sentence beginning on the first line of [Gilmer and Livingston 1992a, page 486]) deferred to a future publication, but now retract. Note that the locations of the irrational jumps $a / 2, a$, and $(1-a) / 2$ in the first half interval together with 1 are linearly dependent over $\mathbb{Q}$. Our next theorem says that this is necessary for the jumps of a signature function which satisfies the $m$-signature averaging condition.

For $0<a<1$, let $\chi_{a}$ denote the characteristic function taking the value 1 on $[0, a)$, value $\frac{1}{2}$ at $a$ and value 0 on $(a, 1]$. We have

$$
\Sigma_{p}\left(\chi_{a}\right)= \begin{cases}\lfloor p a\rfloor & \text { if } p a \notin \mathbb{Z}, \\ \lfloor p a\rfloor-\frac{1}{2} & \text { if } p a \in \mathbb{Z},\end{cases}
$$

where $\lfloor x\rfloor$ denotes the greatest integer in $x$.

For $0<a<\frac{1}{2}$, consider the symmetric jump function $S_{a}=\chi_{1-a}-\chi_{a}$ on $[0,1]$. Then $S_{a} \in \mathscr{Y}$ and

$$
\Sigma_{p}\left(S_{a}\right)=\lfloor p(1-a)\rfloor-\lfloor p a\rfloor .
$$

We define $F_{p}(a)$ by

$$
\Sigma_{p}\left(S_{a}\right)-p \int_{0}^{1} S_{a}(x) d x=F_{p}(a)= \begin{cases}2\langle p a\rangle-1 & \text { if } p a \notin \mathbb{Z}, \\ 0 & \text { if } p a \in \mathbb{Z},\end{cases}
$$

where $\langle x\rangle=x-\lfloor x\rfloor$ denotes the fractional part of $x$.

Theorem 20. Let $\sigma \in \mathscr{S}$ and let $\left\{j_{1}, \ldots, j_{s}\right\}$ be the irrational points of discontinuity of $\sigma$ that lie in the interval $\left[0, \frac{1}{2}\right]$. Suppose $s \geq 1$. If $\sigma$ satisfies the $m$-signature averaging condition, then $\left\{j_{1}, \ldots, j_{s}, 1\right\}$ are linearly dependent over $\mathbb{Q}$.

Proof. It is easily seen that the integral of $\sigma$ must be zero. We assume that there is a jump at an irrational point. Thus $s \geq 1$.

We have that $\sigma$ can be written uniquely as $\sum_{i=1}^{r} c_{i} S_{a_{i}}$ with the $c_{i}$ nonzero and the $a_{i}$ distinct. By reordering, we can assume that $a_{i}$ is rational if and only if $i>s$, for some $s \leq r$. Thus $\left\{j_{1}, \ldots, j_{s}\right\}=\left\{a_{1}, \ldots, a_{s}\right\}$. For each $i>s$, write $a_{i}=b_{i} / d_{i}$ in lowest terms. Let $D$ be the least common multiple of the elements of $\left\{d_{i} \mid i>s\right\} \cup\{m, m+1\}$. Let $N=\{p \mid p>0, p \equiv-1(\bmod D)\}$. For all $p \in N$, $\Sigma_{p} \sigma=0$, and $p a_{i} \notin \mathbb{Z}$. Hence, using (5-1), we have that $\sum_{i=1}^{r} c_{i}\left\langle p a_{i}\right\rangle=r / 2$ for all $p \in N$.

Since $p \in N$ is constant modulo $D, \sum_{i=s+1}^{r} c_{i}\left\langle p a_{i}\right\rangle$ is constant for $p \in N$. Hence the sum over the irrational terms, $\sum_{i=1}^{s} c_{i}\left\langle p a_{i}\right\rangle$ is constant for $p \in N$, as well. Thus

$$
\mathscr{I}=\left\{\left(\left\langle p a_{1}\right\rangle,\left\langle p a_{2}\right\rangle, \ldots,\left\langle p a_{s}\right\rangle\right) \mid p \in N\right\}
$$

is not dense in $I^{s}$. Kronecker's Theorem [Hardy and Wright 1938, Theorem 442] states that if the fractional parts of the positive integral multiples of a vector $\left(a_{1}, a_{2}, \ldots, a_{s}\right)$ are not dense in $I^{s}$, then $\left\{a_{1}, \ldots, a_{s}, 1\right\}$ are linearly dependent over 
$\mathbb{Q}$. It is not hard to see that the same holds for multiples by any arithmetic sequence, like $N$.

The above theorem still holds if one relaxes the hypothesis by removing the condition that the value of $\sigma$ at the jump points be given by the average of the one sided limits, as one could redefine the values at these points without changing the values of $\Sigma_{p}(\sigma)$ for the specified $p$ 's.

Note that, if $a$ is a rational whose denominator divides $d$, then

$$
F_{p}(a)=F_{p+k d}(a)=-F_{-p+k d}(a) .
$$

Definition 21. Given an odd number $d>1$, let $\mathbb{D}(d)$ be the determinant of the $(d-1) / 2 \times(d-1) / 2$ matrix indexed by $1 \leq i, j \leq(d-1) / 2$ with entries

$$
F_{i}(j / d)= \begin{cases}2\langle i j / d\rangle-1 & \text { if } d \nmid i j, \\ 0 & \text { if } d \mid i j .\end{cases}
$$

Conjecture 22. For all odd numbers $d>1, \mathbb{D}(d) \neq 0$.

This conjecture is true for $d$ prime according to the next proposition. We have verified the conjecture for $d<1500$ using Mathematica.

Proposition 23. If $s$ is an odd prime, then $\mathbb{D}(s)= \pm 2^{(s-3) / 2} h_{s} / s$, where $h_{s}$ is the first factor of the class number of the cyclotomic ring $\mathbb{Z}\left[\zeta_{s}\right]$. Thus $\mathbb{D}(s) \neq 0$.

Proof.

The result follows from Equations (1.7), (2.3), (2.4), and (2.5) of [Carlitz and Olson 1955].

Theorem 24. Let $d>1$ be a fixed odd integer for which $\mathbb{D}(d) \neq 0$. Suppose $\sigma \in \mathscr{Y}$ has all jumps at rational points whose denominator divides $d$. If $\Sigma_{p}(\sigma)=0$ for all odd $p$, then $\sigma=0$.

Proof. We have $\sigma=\sum_{j=1}^{(d-1) / 2} a_{j} S_{j / d}$ for some $a_{j}$. Since $\Sigma_{p}(\sigma)=0$ for all odd $p$, we have $\int_{0}^{1} \sigma(x) d x=0$. We pick an odd integer $p(i)$ congruent to $i$ modulo $p$ for every $i$ in the range $0 \leq i \leq(d-1) / 2$. For each $i$, we have $\Sigma_{p(i)}(\sigma)-p(i) \int_{0}^{1} \sigma(x) d x=0$. Using Equations (5-1) and (5-2), this gives us the linear equation $\sum_{j=1}^{(d-1) / 2} a_{j} F_{i}(j / d)=0$. The resulting system of $(d-1) / 2$ equations in the $(d-1) / 2$ unknowns $a_{j}$ has only the trivial solution if $\mathbb{D}(d) \neq 0$.

Corollary 25. Suppose $d>1$ is an odd integer and $\mathbb{D}(d) \neq 0$. A nonzero knot signature function satisfying the 1-signature averaging condition cannot have jumps only at points whose denominator is a divisor of $d$.

Since knot signature functions cannot jump at points with prime denominators [Tristram 1969], Proposition 23 does not say anything about knots, except to the extent that it makes Conjecture 22 plausible. 


\section{Appendix A: Witt invariants of cable knots}

The proof of Theorem 15 follows fairly readily from work of Litherland, some basic knot theoretic results, and consideration of Witt groups.

We begin with an observation: if $\mathbb{S}$ is a satellite of $K$ with orbit $P$ and winding number $n$, then for an appropriate choice of Seifert surfaces for $K, P$, and $\mathbb{S}$, the Seifert matrix for $\mathbb{S}$ is the direct sum of a Seifert matrix for $P$ and one for $C_{n, 1}$. The construction of the Seifert surfaces for a satellite knot, which leads to the above result, goes back to [Seifert 1950].

Thus, to prove Theorem 15 we need only prove the following:

Theorem 26. For $C_{(n, 1)}$, the $(n, 1)$-cable of $C$,

$$
w_{C_{(n, 1)}}(j / p)=w_{C}(n j / p) .
$$

Proof. The proof is largely contained in a diagram; note in the following description that the central square of the diagram is not apparently commutative, while one has commutativity around the other interior faces of the diagram.

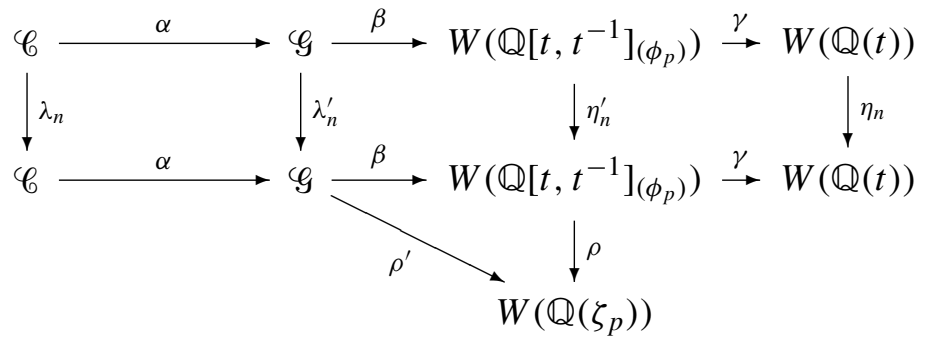

Here is the notation and necessary background:

- $\mathscr{C}$ is the concordance group; $\mathscr{G}$ is Levine's algebraic concordance group of Seifert matrices; $\alpha$ is the homomorphism induced by $K \rightarrow V_{K}$.

- $W\left(\mathbb{Q}\left[t, t^{-1}\right]_{\left(\phi_{p}\right)}\right.$ is the Witt group of the localization of $\mathbb{Q}\left[t, t^{-1}\right]$ at the $p$ cyclotomic polynomial $\phi_{p}$ (that is, the domain formed by inverting all polynomials relatively prime to $\phi_{p}$ ); $\beta$ is the map induced by

$$
V \rightarrow(1-t) V+\left(1-t^{-1}\right) V^{t} .
$$

- $W(\mathbb{Q}(t))$ is the Witt group of the field of fractions of $\mathbb{Q}\left[t, t^{-1}\right] ; \gamma$ is induced by inclusion. The inclusion map is injective (see [Milnor and Husemoller 1973, Corollary IV 3.3] in the symmetric case, and [Ranicki 1981, Proposition 4.2.1 iii] for the hermitian case that arises here).

- $\lambda_{n}$ is the function induced by forming the $(n, 1)$-cable; $\lambda_{n}^{\prime}$ is the homomorphism induced by $\lambda_{n}$. This map can be given explicitly in terms of Seifert matrices. That this induces a map on $\mathscr{G}$ and that the map is a homomorphism is elementary (see [Cha et al. 2008; Kawauchi 1980] for further discussion). 
- The map $\rho$ is induced by the map $t \rightarrow \zeta_{p}$.

- The map $\eta_{n}$ (respectively $\eta_{n}^{\prime}$ ) is induced by the embedding of $\mathbb{Q}(t)$ (respectively $\left.\mathbb{Q}\left[t, t^{-1}\right]_{\left(\phi_{p}\right)}\right)$ into itself which sends $t$ to $t^{n}$.

The proof of Theorem 15 is seen to be equivalent to showing that

$$
\rho^{\prime} \circ \alpha \circ \lambda_{n}=\rho \circ \eta_{n}^{\prime} \circ \beta \circ \alpha .
$$

By writing $\rho^{\prime}=\rho \circ \beta$, we see this will follow from

$$
\beta \circ \alpha \circ \lambda_{n}=\eta_{n}^{\prime} \circ \beta \circ \alpha .
$$

According to Litherland [1984], we have

$$
\gamma \circ \beta \circ \alpha \circ \lambda_{n}=\eta_{n} \circ \gamma \circ \beta \circ \alpha .
$$

Using commutativity of the rightmost square, we have $\eta_{n} \circ \gamma=\gamma \circ \eta_{n}^{\prime}$, so Litherland's equality can be rewritten as

$$
\gamma \circ \beta \circ \alpha \circ \lambda_{n}=\gamma \circ \eta_{n}^{\prime} \circ \beta \circ \alpha .
$$

Finally, because $\gamma$ is injective, this implies $\beta \circ \alpha \circ \lambda_{n}=\eta_{n}^{\prime} \circ \beta \circ \alpha$, as desired.

\section{Appendix B: One approach to Theorem 17}

Let $\mathbb{Q}^{\prime}=\left\{r / s \in \mathbb{Q} \mid \operatorname{gcd}(s, r)=\operatorname{gcd}\left(s,\left|\operatorname{Torsion}\left(H_{1}(R)\right)\right|\right)=1\right\}$.

Lemma 27. If $T$ is a finitely generated torsion group, and the prime divisors of $|T|$ are all divisors of $\left|\operatorname{Torsion}\left(H_{1}(R)\right)\right|$, then $T \otimes\left(\mathbb{Q}^{\prime} / \mathbb{Z}\right)=0$, and $\operatorname{Tor}\left(T, \mathbb{Q}^{\prime} / \mathbb{Z}\right)=0$. Proof. It suffices to prove this for $T$ a finite cyclic group of order $k$ relatively prime to all the denominators of elements of $\mathbb{Q}^{\prime}$. From the short exact sequence

$$
0 \rightarrow \mathbb{Z} \stackrel{k}{\rightarrow} \mathbb{Z} \rightarrow T \rightarrow 0,
$$

we obtain

$$
0 \rightarrow \operatorname{Tor}\left(T, \mathbb{Q}^{\prime} / \mathbb{Z}\right) \rightarrow \mathbb{Q}^{\prime} / \mathbb{Z} \stackrel{k^{\prime}}{\rightarrow} \mathbb{Q}^{\prime} / \mathbb{Z} \rightarrow T \otimes \mathbb{Q}^{\prime} / \mathbb{Z} \rightarrow 0 .
$$

Suppose $s$ is a denominator of an element in $\mathbb{Q}^{\prime}$; then $\operatorname{gcd}(k, s)=1$, so there exists $a, b \in \mathbb{Z}$ such that $k a+s b=1$. It follows that $k \cdot a / s \equiv 1 / s(\bmod 1)$. Thus $k \cdot: \mathbb{Q}^{\prime} / \mathbb{Z} \rightarrow \mathbb{Q}^{\prime} / \mathbb{Z}$ is surjective. It is easy to see that it is also injective.

Lemma 28. A short exact sequence of the form

$$
0 \rightarrow T_{1} \stackrel{\psi}{\rightarrow} T_{2} \oplus F_{2} \stackrel{\phi}{\rightarrow} T_{3} \oplus F_{3} \rightarrow 0
$$

where the $F_{i}$ are free abelian groups and the $T_{i}$ are torsion groups, induces a short exact sequence

$$
0 \rightarrow T_{1} \stackrel{\pi_{T_{2}} \circ \psi}{\longrightarrow} T_{2} \stackrel{\phi_{\mid T_{2}}}{\longrightarrow} T_{3} \rightarrow 0 .
$$


Proof. Exactness on the left and at the middle of this sequence is immediate. We only need to show that $\phi_{\mid T_{2}}$ is surjective. Let $x \in T_{3}$; there exist $(y, z) \in T_{2} \oplus F_{2}$ with $\phi((y, z))=x$. We wish to show that $z=0$. There exist nonzero integers $n$ and $m$ such that $n x=0$ and $m y=0$. Then $\phi((0, m n z))=\phi((m n y, m n z))=m n x=0$. By exactness of the original sequence, $(0, m n z) \in \psi\left(T_{1}\right)$. Since $z \in F_{2}$, we have that $z=0$.

Lemma 29. If $\mathscr{T}=$ Torsion $\left(H_{1}(R)\right)$ and $H$ denotes the kernel of $H_{1}(F) \rightarrow H_{1}(R) / \mathscr{T}$, then $H \otimes \mathbb{Q}^{\prime} / \mathbb{Z}$ is the kernel of the natural map $H_{1}(F) \otimes\left(\mathbb{Q}^{\prime} / \mathbb{Z}\right) \rightarrow H_{1}(R) \otimes\left(\mathbb{Q}^{\prime} / \mathbb{Z}\right)$.

Proof. Let $\mathscr{I}$ and $\hat{\mathscr{I}}$ be the images of $H_{1}(F) \rightarrow H_{1}(R)$ and $H_{1}(F) \rightarrow H_{1}(R) / \mathcal{T}$, respectively. We have a short exact sequence

$$
0 \rightarrow H \rightarrow H_{1}(F) \rightarrow \hat{\Phi} \rightarrow 0 .
$$

As $\hat{\mathscr{\Phi}}$ is free abelian, $\operatorname{Tor}\left(\hat{\mathscr{\Phi}}, \mathbb{Q}^{\prime} / \mathbb{Z}\right)=0$, and we then have a short exact sequence

$$
0 \rightarrow H \otimes\left(\mathbb{Q}^{\prime} / \mathbb{Z}\right) \rightarrow H_{1}(F) \otimes\left(\mathbb{Q}^{\prime} / \mathbb{Z}\right) \rightarrow \hat{\Phi} \otimes\left(\mathbb{Q}^{\prime} / \mathbb{Z}\right) \rightarrow 0 .
$$

Let $\mathscr{R}$ denote $H_{1}(R)$, and note that $\mathscr{I} /(\mathscr{T} \cap \mathscr{T})=\hat{\mathscr{J}}$. Consider the lattice of subgroups consisting of $\mathscr{R}, \mathscr{I}, \mathscr{T}$, and $\mathscr{I} \cap \mathscr{T}$. Their inclusions fit into the following commutative diagram with exact rows and columns:

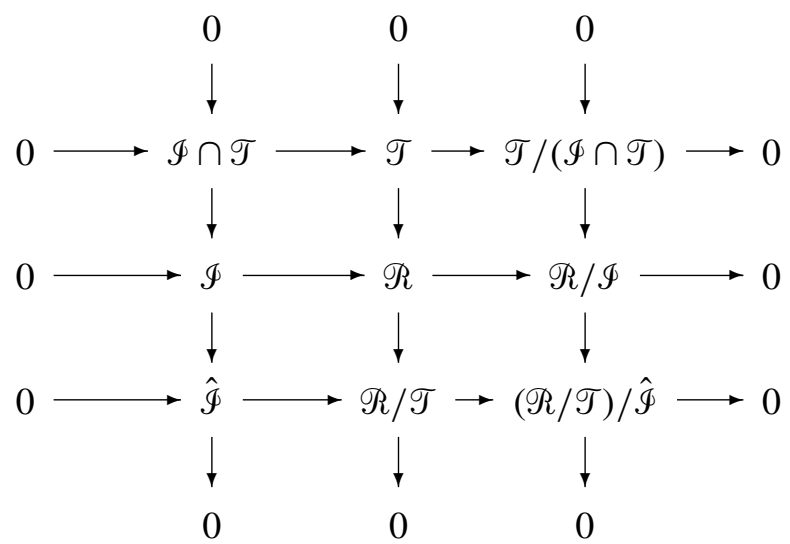

To see exactness, view the first two columns as the inclusion of one chain complex into another. The third column is the quotient chain complex. Thus we have a short exact sequence of chain complexes. The first two chain complexes are clearly exact. It follows that the third column is exact, using the associated long exact sequence of homology groups.

Using the long exact sequence of the pair $(R, F)$, we may identify $\mathscr{R} / \mathscr{I}$ with $H_{1}(R, F)$. Using Lefschetz duality and the universal coefficient theorem, we have $H_{1}(R, F) \approx H^{2}(R, \Delta) \approx H^{2}(R) \approx \mathscr{T} \oplus \mathbb{Z}^{\beta_{2}(R)}$. With these identifications, the last 
column of the diagram becomes a short exact sequence

$$
0 \rightarrow \mathscr{T} /(\mathscr{T} \cap \mathscr{T}) \rightarrow \mathscr{T} \oplus \mathscr{F} \rightarrow \operatorname{Torsion}((\mathscr{R} / \mathscr{T}) / \hat{\mathscr{F}}) \oplus \mathscr{F}^{\prime} \rightarrow 0,
$$

where $\mathscr{F}_{F}$ and $\mathscr{F}^{\prime}$ are free abelian groups. By Lemma 28, there is a short exact sequence

$$
0 \rightarrow \mathscr{T} /(\mathscr{I} \cap \mathscr{T}) \rightarrow \mathscr{T} \rightarrow \operatorname{Torsion}((\mathscr{R} / \mathscr{T}) / \hat{\mathscr{T}}) \rightarrow 0 .
$$

We conclude that $\mid \operatorname{Torsion}(\mathscr{R} / \mathscr{T}) / \hat{\mathscr{T}})|=| \mathscr{I} \cap \mathscr{T} \mid$. By Lemma 27, we have

$$
\operatorname{Tor}\left((\mathscr{R} / \mathscr{T}) / \hat{\mathscr{T}}, \mathbb{Q}^{\prime} / \mathbb{Z}\right)=\operatorname{Tor}\left(\operatorname{Torsion}((\mathscr{R} / \mathscr{T}) / \hat{\mathscr{T}}), \mathbb{Q}^{\prime} / \mathbb{Z}\right)=0,
$$

so the sequence obtained from the last row of the diagram upon tensoring with $\mathbb{Q}^{\prime} / \mathbb{Z}$ is exact. In particular, the map $\hat{\Phi} \otimes\left(\mathbb{Q}^{\prime} / \mathbb{Z}\right) \rightarrow\left(H_{1}(R) / \mathscr{T}\right) \otimes\left(\mathbb{Q}^{\prime} / \mathbb{Z}\right)$ is injective. It follows that $H \otimes\left(\mathbb{Q}^{\prime} / \mathbb{Z}\right)$, the kernel of $H_{1}(F) \otimes\left(\mathbb{Q}^{\prime} / \mathbb{Z}\right) \rightarrow \hat{\Phi} \otimes\left(\mathbb{Q}^{\prime} / \mathbb{Z}\right)$, is the same as the kernel of $H_{1}(F) \otimes\left(\mathbb{Q}^{\prime} / \mathbb{Z}\right) \rightarrow\left(H_{1}(R) / \mathscr{T}\right) \otimes\left(\mathbb{Q}^{\prime} / \mathbb{Z}\right)$.

Considering the middle column, we obtain the following exact sequence:

$$
\mathscr{T} \otimes\left(\mathbb{Q}^{\prime} / \mathbb{Z}\right) \rightarrow H_{1}(R) \otimes\left(\mathbb{Q}^{\prime} / \mathbb{Z}\right) \rightarrow H_{1}(R) / \mathscr{T} \otimes\left(\mathbb{Q}^{\prime} / \mathbb{Z}\right) \rightarrow 0 .
$$

Since $\mathscr{T} \otimes\left(\mathbb{Q}^{\prime} / \mathbb{Z}\right)=0$ by Lemma 27 , we see that

$$
H_{1}(R) \otimes\left(\mathbb{Q}^{\prime} / \mathbb{Z}\right) \rightarrow\left(H_{1}(R) / \mathscr{T}\right) \otimes\left(\mathbb{Q}^{\prime} / \mathbb{Z}\right)
$$

is injective. Thus the kernel of $H_{1}(F) \otimes\left(\mathbb{Q}^{\prime} / \mathbb{Z}\right) \rightarrow\left(H_{1}(R) / \mathscr{T}\right) \otimes\left(\mathbb{Q}^{\prime} / \mathbb{Z}\right)$ is also the kernel of $H_{1}(F) \otimes\left(\mathbb{Q}^{\prime} / \mathbb{Z}\right) \rightarrow H_{1}(R) \otimes\left(\mathbb{Q}^{\prime} / \mathbb{Z}\right)$.

The second to last sentence of [Gilmer 1993, page 6] asserts without justification, in the situation of Lemma 29 , that $H \otimes \mathbb{Q} / \mathbb{Z}$ is the kernel of the natural map $H_{1}(F) \otimes(\mathbb{Q} / \mathbb{Z}) \rightarrow H_{1}(R) \otimes(\mathbb{Q} / \mathbb{Z})$. The original proof of the theorem may then be modified using Lemma 29 and replacing $\mathbb{Q} / \mathbb{Z}$ by $\mathbb{Q}^{\prime} / \mathbb{Z}$ judiciously. This proof then yields the conclusion: $A^{q}{ }_{p} \cap(H \otimes \mathbb{Q} / \mathbb{Z})$ (in the notation of [Gilmer 1993]) is equal to $\mathfrak{m}(q, \Delta)_{(p)}$ for primes $p$ relatively prime to $\mid \operatorname{Torsion}\left(H_{1}(R) \mid\right.$. This, in turn, can be rephrased as Theorem 17 .

\section{Appendix C: Another approach to Theorem 17}

\section{C.1. Notation.}

- $K$ is a slice knot with a genus $g$ Seifert surface $F ; K$ bounds a slice disk $\Delta$; $R \subset B^{4}$ is a 3-manifold bounded by $F \cup \Delta$.

- $S_{q}$ is the $q$-fold branched cover of $S^{3}$ branched over $K ; W_{q}$ is the $q$-fold branched cover of $B^{4}$ branched over $\Delta$.

- $H$ is the kernel of $H_{1}(F) \rightarrow H_{1}(R) / \operatorname{Torsion}\left(H_{1}(R)\right) ; \kappa(q, \Delta)$ is the kernel of $H_{1}\left(S_{q}\right) \rightarrow H_{1}\left(W_{q}\right)$. 
We further choose generators for various homology groups:

- $\left\{x_{i}^{\prime}\right\} \cup\left\{y_{i}^{\prime}\right\}$ is a symplectic basis of $H_{1}(F)$ where the $x_{i}$ generate $H$.

- $F$ is built from a disk with 1-handles added corresponding to this basis. The dual linking circles to the bands represent homology classes in $H_{1}\left(S^{3} \backslash F\right)$ denoted $\left\{x_{i}\right\} \cup\left\{y_{i}\right\}$.

Recall (see [Rolfsen 1976]) that $S_{q}$ is built from $q$ copies of $S^{3} \backslash F$. These copies can be enumerated cyclically, corresponding to translates under the deck transformation. There is a corresponding enumeration of the lifts of $F$ to $S_{q}$.

- The lifts of the $x_{i}$ are denoted $\tilde{x}_{i, \alpha}$, and similarly for the $\tilde{y}_{i}, \tilde{x}_{i}^{\prime}$ and $\tilde{y}_{i}^{\prime}$. The $\alpha$ are indices denoting the appropriate lift of $S^{3} \backslash F$ and $F$. Here, $\alpha \in \mathbb{Z}_{q}$.

- Yy denotes the subgroup of $H_{1}\left(S_{q}\right)$ generated by the $\tilde{y}_{i, \alpha}$. Similarly for $\mathscr{X}, \mathcal{Y}^{\prime}$, and $\mathscr{X}^{\prime}$.

- $\mathbb{Y}$ denotes the subgroup of $H_{1}\left(S_{q}\right)$ generated by a single set of lifts $\left\{\tilde{y}_{i, 0}\right\}$.

C.2. Statement and proof summary. Theorem 17 can now be stated succinctly: if $p$ is relatively prime to the order of $\operatorname{Torsion}\left(H_{1}(R)\right)$, then $\kappa(\Delta, q)_{(p)}=\mathbb{Y}_{(p)}$. The proof has several steps:

- Lemma 30: $H_{1}\left(S_{q}\right)_{(p)}=\mathscr{Y}_{(p)} \oplus \mathscr{X}_{(p)}$ and $\left|\mathscr{Y}_{(p)}\right|=\left|\mathscr{X}_{(p)}\right|$.

- Lemma 31: $\mathscr{X}_{(p)}^{\prime}=\mathscr{Y}_{(p)}$.

- Lemma 32: $\mathscr{X}_{(p)}^{\prime} \subset \kappa(\Delta, q)_{(p)}$ and $\left|\kappa(\Delta, q)_{(p)}\right|^{2}=\left|H_{1}\left(S_{q}\right)_{(p)}\right|$.

- Lemma 33: $\mathbb{Y}_{(p)}=\mathscr{Y}_{(p)}$.

Proof of Theorem 17. We want to show that $\kappa(\Delta, q)_{(p)}=\mathbb{Y}_{(p)}$. By Lemma 33, this is equivalent to showing that $\kappa(\Delta, q)_{(p)}=\mathscr{Y}_{(p)}$. By Lemmas 30 and 32, the orders of these two groups are the same. By Lemmas 31 and $32, \mathscr{Y}_{(p)} \subset \kappa(\Delta, q)$, and the proof is complete.

\section{C.3. Proofs of lemmas.}

Lemma 30. $H_{1}\left(S_{q}\right)_{(p)}=\mathscr{Y}_{(p)} \oplus \mathscr{X}_{(p)}$ and $\left|\mathscr{Y}_{(p)}\right|=\left|\mathscr{X}_{(p)}\right|$.

Proof. We use the convention that the Seifert form $V$ is the pairing $V(a, b)=$ $\operatorname{link}\left(i_{+}(a), b\right)$, where $i_{+}$is the positive push-off. For transformations, we have matrices acting on the left; in presentation matrices, the rows give the relations.

The Seifert matrix of $V$ for the surface $F$ with respect to the basis $\left\{x_{i}^{\prime}\right\} \cup\left\{y_{i}^{\prime}\right\}$ for $H_{1}(F)$ is of the form

$$
\left(\begin{array}{cc}
0 & M \\
M^{t}+I & B
\end{array}\right)
$$

for some $g$ dimensional square matrices $M$ and $B$, with $B$ symmetric. 
The first homology of $S_{q}$ is generated by (all) the lifts of the $x_{i}$ and $y_{i}$, which we have denoted $\tilde{x}_{i, \alpha}$ and $\tilde{y}_{i, \alpha}$. As described, for instance in [Rolfsen 1976, page 213], a presentation matrix of the first homology of $S_{q}$ with respect to this basis is determined by $V$. In this case, the result is a matrix of the form

$$
\left(\begin{array}{cc}
0 & M \\
M^{\prime} & \mathscr{B}
\end{array}\right)
$$

where $M$ and $\mathscr{B}$ are $q g$ dimensional matrices that are built out of the blocks of $V$ as follows (we illustrate in the case $q=3$ ):

$$
\begin{gathered}
\mathcal{M =}\left(\begin{array}{ccc}
M+I & -M & 0 \\
0 & M+I & -M \\
-M & 0 & M+I
\end{array}\right), \quad \mathcal{M}^{\prime}=\left(\begin{array}{ccc}
M^{t} & -M^{t}-I & 0 \\
0 & M^{t} & -M^{t}-I \\
-M^{t}-I & 0 & M^{t}
\end{array}\right), \\
\mathscr{B}=\left(\begin{array}{ccc}
B & -B & 0 \\
0 & B & -B \\
-B & 0 & B
\end{array}\right) .
\end{gathered}
$$

The first columns correspond to the $\tilde{x}_{i, \alpha}$ and the later columns to the $\tilde{y}_{i, \alpha}$.

Notice first that $|\mathcal{M}|=\left|\mathcal{M}^{\prime}\right|$ and $|\mathcal{M}|^{2}=\left|H_{1}\left(S_{q}\right)\right|$.

Forming the quotient, $H_{1}\left(S_{q}\right) / \mathscr{Y}$ yields a group $\overline{\mathscr{X}}$ generated by the image of $\mathscr{X}$. This quotient is presented by $\mathcal{M}^{\prime}$, and thus has order $\sqrt{\left|H_{1}\left(S_{q}\right)\right|}$, so $\mathscr{X}$ has order at least this large. Thus $\left|\mathscr{X}_{(p)}\right|^{2} \geq\left|H_{1}\left(S_{q}\right)_{(p)}\right|$. On the other hand, since $\mathscr{X}_{(p)}$ is a self-annihilating subgroup for a nonsingular form, $\left|\mathscr{X}_{(p)}\right|^{2} \leq\left|H_{1}\left(S_{q}\right)_{(p)}\right|$.

We now have $\left|\mathscr{X}_{(p)}\right|^{2}=\left|H_{1}\left(S_{q}\right)_{(p)}\right|$, and thus $\left|\mathscr{X}_{(p)}\right|=\left|\overline{\mathscr{X}}_{(p)}\right|$. From this we can conclude that $\mathscr{X}_{p} \cap \mathscr{Y}_{(p)}=0$, so $H_{1}\left(S_{q}\right)_{(p)}=\mathscr{X}_{(p)} \oplus \mathscr{Y}_{(p)}$.

Lemma 31. $\mathscr{X}_{(p)}^{\prime}=\mathscr{Y}_{(p)}$.

Proof. The positive and negative push-off maps $i_{ \pm}: H_{1}(F) \rightarrow S^{3} \backslash F$ send the span of the $x_{i}^{\prime}$ to the span of the $y_{i}$. Denote the restriction of these maps by $j_{ \pm}:\left\langle\left\{x_{i}^{\prime}\right\}\right\rangle \rightarrow\left\langle\left\{y_{i}\right\}\right\rangle$. With respect to these bases, the maps $j_{ \pm}$are given by the matrices $M^{t}$ and $M^{t}+I$. Now view these matrices as defining maps from $\mathbb{Z}^{g}$ to itself with $M^{t}$ corresponding to an automorphism $T$. Then any element $y \in \mathbb{Z}^{g}$ can be written $y=\operatorname{Id}(y)=(T+\operatorname{Id})(y)-T(y)$. Thus, Image $\left(j_{+}\right)+\operatorname{Image}\left(j_{-}\right)=\operatorname{Span}\left(\left\{y_{i}\right\}\right)$. Lifting to the $q$-fold branched covers, we see that the $\tilde{y}_{i, \alpha}$ are all in the image of the $\tilde{x}_{i, \alpha}^{\prime}$ (in more detail, each $\tilde{y}_{i, \alpha}$ is in the span of the images of the $\left\{\tilde{x}_{i, \alpha}^{\prime}\right\}$ and $\left\{\tilde{x}_{i, \alpha+1}^{\prime}\right\}$ ). Also, the images of the $\tilde{x}_{i, \alpha}^{\prime}$ are all in $\operatorname{Span}\left(\left\{\tilde{y}_{i, \alpha}\right\}\right)$. The same thus holds on the level of the $p$-torsion, completing the proof of the lemma.

Lemma 32. $\mathscr{X}_{(p)}^{\prime} \subset \kappa(\Delta, q)_{(p)}$ and $\left|\kappa(\Delta, q)_{(p)}\right|^{2}=\left|H_{1}\left(S_{q}\right)_{(p)}\right|$.

Proof. Let $\gamma=\left|\operatorname{Torsion}\left(H_{1}(R)\right)\right|$. Then $\gamma z=0 \in H_{1}(R)$ for all $z \in H$. Lifting, we see that $z^{\prime} \in \mathscr{X}_{(p)}^{\prime}$ for all $\gamma z^{\prime}=0 \in H_{1}\left(W_{q}\right)$, so $\gamma \mathscr{X}_{(p)}^{\prime} \subset \kappa(\Delta, q)_{(p)}$. But multiplication by $\gamma$ is an isomorphism on $\mathscr{X}_{(p)}^{\prime}$ since $p$ is relatively prime to $\gamma$. 
We have from Theorem 16 that $|\kappa(\Delta, q)|^{2}=\left|H_{1}\left(S_{q}\right)\right|$, so the same holds for the $p$-torsion.

Lemma 33. $\mathbb{Y}_{(p)}=\mathscr{Y}_{(p)}$.

Proof. Let $\Lambda=\mathbb{Z}\left[\mathbb{Z}_{q}\right]$, the group ring of the cyclic group. We write $\mathbb{Z}_{q}$ multiplicatively, generated by $t$. The standard derivation of a presentation of the homology $H_{1}\left(S_{q}\right)$, such as in [Rolfsen 1976], is a Mayer-Vietoris argument. The homology groups involved are all modules over $\Lambda$, where $t$ acts by the deck transformation. From this viewpoint, the Mayer-Vietoris sequence now yields that as a $\Lambda$-module the homology is given as a quotient $H_{1}\left(S_{q}\right) \cong \Lambda^{2 g} /\left(V-t V^{t}\right) \Lambda^{2 g}$.

Since $V-V^{t}$ is invertible, we can multiply the quotienting submodule by $\left(V-V^{t}\right)^{-1}$ without changing the quotient space. Some elementary algebra then shows that

$$
H_{1}\left(S_{q}\right) \cong \Lambda^{2 g} /(\Gamma+t(I-\Gamma)) \Lambda^{2 g},
$$

where $\Gamma=\left(V-V^{t}\right)^{-1} V$.

It is clear from this that for any $z \in \Lambda^{2 g}$, we have $\Gamma z=t(\Gamma-I) z \in H_{1}\left(S_{q}\right)$. Thus, $\Gamma^{q} z=t^{q}(\Gamma-I)^{q} z \in H_{1}\left(S_{q}\right)$. However, $t^{q}=1$, so $\Gamma^{q}-(\Gamma-I)^{q}$ annihilates $H_{1}\left(S_{q}\right)$.

Expanding, we have that for some polynomial $f$ with constant term 0 and of degree $q-1$, the action of $f(\Gamma)$ on $H_{1}\left(S_{q}\right)$ coincides with $I$. The leading coefficient of $f$ is $q$. If $p$ does not divide the order $\left|H_{1}\left(S_{q}\right)\right|$, the lemma is immediately true, so assume $p$ divides the order $\left|H_{1}\left(S_{q}\right)\right|$. We know that $p$ is relatively prime to $q$. Thus, we can switch to $\mathbb{Z}_{(p)}$-coefficients, in which case the leading coefficient of $f$ is a unit, and we see that with $\mathbb{Z}_{(p)}$-coefficients, $\Gamma$ is invertible.

We now focus on the Seifert matrix $V$ of the algebraically slice knot. In the coordinates we have been using, we see that

$$
\Gamma=\left(\begin{array}{cc}
M^{t}+I & B \\
0 & -M
\end{array}\right) .
$$

From this we conclude that with $\mathbb{Z}_{(p)}$-coefficients, $M$ and $M+I$ are both invertible. Recall that for each $k, M$ and $M+I$ determine the maps from $\operatorname{Span}\left(\tilde{x}_{i, k}^{\prime}\right)$ and $\operatorname{Span}\left(\tilde{x}_{i, k+1}^{\prime}\right)$ to $\operatorname{Span}\left(\tilde{y}_{i, k}\right)$. Thus, any element in $\operatorname{Span}\left(\tilde{y}_{i, k}\right)$ is also in $\operatorname{Span}\left(\tilde{y}_{i, k+1}\right)$. This completes the proof of the lemma.

\section{Acknowledgments}

We thank John Ewing for his help with the number theoretic aspects of this project. In particular, John pointed out to us the relationship between the determinant $\mathbb{D}(s)$ and class numbers. We also appreciate Stefan Friedl's careful reading and thoughtful commentary on our initial draft of this article. 


\section{References}

[Carlitz and Olson 1955] L. Carlitz and F. R. Olson, "Maillet's determinant", Proc. Amer. Math. Soc. 6:2 (1955), 265-269. MR 16,999d Zbl 0065.02703

[Casson and Gordon 1978] A. Casson and C. M. Gordon, Lecture given by C. McA. Gordon at Cambridge University, 1978.

[Casson and Gordon 1986] A. J. Casson and C. M. Gordon, "Cobordism of classical knots", pp. 181-199 in À la recherche de la topologie perdue, edited by L. Guillou and A. Marin, Progr. Math. 62, Birkhäuser, Boston, MA, 1986. MR 900252 Zbl 0597.57001

[Cha and Livingston 2011] J. C. Cha and C. Livingston, "KnotInfo: Table of knot invariants", 2011, Available at http://www.indiana.edu/ knotinfo.

[Cha et al. 2008] J. C. Cha, C. Livingston, and D. Ruberman, "Algebraic and Heegaard-Floer invariants of knots with slice Bing doubles", Math. Proc. Cambridge Philos. Soc. 144:2 (2008), 403-410. MR 2009h:57013 Zbl 1144.57002

[Cochran et al. 2003] T. D. Cochran, K. E. Orr, and P. Teichner, "Knot concordance, Whitney towers and $L^{2}$-signatures", Ann. of Math. (2) 157:2 (2003), 433-519. MR 2004i:57003 Zbl 1044.57001

[Cooper 1982] D. Cooper, Signatures of surfaces with applications to knot and link cobordism, Ph.D. thesis, University of Warwick, 1982, Available at http://library.msri.org/books/cooper.

[Freedman and Quinn 1990] M. H. Freedman and F. Quinn, Topology of 4-manifolds, Princeton Mathematical Series 39, Princeton University Press, 1990. MR 94b:57021 Zbl 0705.57001

[Friedl 2004] S. Friedl, "Eta invariants as sliceness obstructions and their relation to Casson-Gordon invariants”, Algebr. Geom. Topol. 4 (2004), 893-934. MR 2005j:57016 Zbl 1067.57003

[Gilmer 1983] P. M. Gilmer, "Slice knots in $S^{3}$ ", Quart. J. Math. Oxford Ser. (2) 34:135 (1983), 305-322. MR 85d:57004 Zbl 0542.57007

[Gilmer 1993] P. Gilmer, "Classical knot and link concordance", Comment. Math. Helv. 68:1 (1993), 1-19. MR 94c:57007 Zbl 0805.57005

[Gilmer and Livingston 1992a] P. Gilmer and C. Livingston, "The Casson-Gordon invariant and link concordance”, Topology 31:3 (1992), 475-492. MR 93h:57037 Zbl 0797.57001

[Gilmer and Livingston 1992b] P. Gilmer and C. Livingston, "Discriminants of Casson-Gordon invariants", Math. Proc. Cambridge Philos. Soc. 112:1 (1992), 127-139. MR 94e:57007 Zbl 0791.57015

[Hardy and Wright 1938] G. H. Hardy and E. M. Wright, An introduction to the theory of numbers, Oxford University Press, 1938. Zbl 0020.29201

[Hedden 2007] M. Hedden, "Knot Floer homology of Whitehead doubles", Geom. Topol. 11 (2007), 2277-2338. MR 2008m:57030 Zbl 1187.57015

[Hedden 2009] M. Hedden, "On knot Floer homology and cabling, II", Int. Math. Res. Not. 2009:12 (2009), 2248-2274. MR 2011f:57015 Zbl 1172.57008

[Kauffman 1987] L. H. Kauffman, On knots, Annals of Mathematics Studies 115, Princeton University Press, 1987. MR 89c:57005 Zbl 0627.57002

[Kawauchi 1980] A. Kawauchi, "On links not cobordant to split links", Topology 19:4 (1980), 321-334. MR 82d:57011 Zbl 0805.57005

[Kirby 1978] R. Kirby, "Problems in low dimensional manifold theory", pp. 273-312 in Algebraic and geometric topology (Stanford, CA, 1976), edited by R. J. Milgram, Proc. Sympos. Pure Math. 32, Amer. Math. Soc., Providence, R.I., 1978. MR 80g:57002 Zbl 0394.57002

[Levine 1969] J. Levine, "Invariants of knot cobordism", Invent. Math. 8 (1969), 98-110. MR 40 \#6563 Zbl 0179.52401 
[Litherland 1984] R. A. Litherland, "Cobordism of satellite knots", pp. 327-362 in Four-manifold theory (Durham, NH, 1982)), edited by C. Gordon and R. Kirby, Contemp. Math. 35, Amer. Math. Soc., Providence, RI, 1984. MR 86k:57003 Zbl 0563.57001

[Livingston and Melvin 1985] C. Livingston and P. Melvin, "Abelian invariants of satellite knots", pp. 217-227 in Geometry and topology (College Park, MD, 1983/84)), edited by J. Alexander and J. Harer, Lecture Notes in Math. 1167, Springer, Berlin, 1985. MR 87e:57009 Zbl 0605.57001

[Milnor and Husemoller 1973] J. Milnor and D. Husemoller, Symmetric bilinear forms, Ergebnisse der Mathematik und ihrer Grenzgebiete 73, Springer, New York, 1973. MR 58 \#22129 Zbl 0292.10016

[Naik 1996] S. Naik, "Casson-Gordon invariants of genus one knots and concordance to reverses", $J$. Knot Theory Ramifications 5:5 (1996), 661-677. MR 97h:57020 Zbl 0890.57014

[Ozsváth and Szabó 2003] P. Ozsváth and Z. Szabó, "Knot Floer homology and the four-ball genus", Geom. Topol. 7 (2003), 615-639. MR 2004i:57036 Zbl 1037.57027

[Ranicki 1981] A. Ranicki, Exact sequences in the algebraic theory of surgery, Mathematical Notes 26, Princeton University Press, 1981. MR 82h:57027 Zbl 0471.57012

[Rolfsen 1976] D. Rolfsen, Knots and links, Mathematics Lecture Series 7, Publish or Perish, Berkeley, 1976. MR 58 \#24236 Zbl 0339.55004

[Seifert 1950] H. Seifert, "On the homology invariants of knots", Quart. J. Math., Oxford Ser. (2) 1 (1950), 23-32. MR 11,735b Zbl 0035.11103

[Tristram 1969] A. G. Tristram, "Some cobordism invariants for links", Proc. Cambridge Philos. Soc. 66 (1969), 251-264. MR 40 \#2104 Zbl 0191.54703

Received September 7, 2012. Revised December 27, 2012.

PATRICK M. GILMER

DEPARTMENT OF MATHEMATICS

LOUISIANA STATE UNIVERSITY

376 LOCKETT HALL

BATON Rouge, LA 70803

UNITED STATES

gilmer@math.lsu.edu

CHARLES LiVINGSTON

DEPARTMENT OF MATHEMATICS

INDIANA UNIVERSITY

RAWLES HALL

BLOOMINGTON, IN 47405-5701

UNITED STATES

livingst@indiana.edu 


\title{
PACIFIC JOURNAL OF MATHEMATICS
}

\author{
msp.org/pjm
}

Founded in 1951 by E. F. Beckenbach (1906-1982) and F. Wolf (1904-1989)

\section{EDITORS}

V. S. Varadarajan (Managing Editor)

Department of Mathematics

University of California

Los Angeles, CA 90095-1555

pacific@math.ucla.edu

Paul Balmer

Department of Mathematics

University of California

Los Angeles, CA 90095-1555

balmer@math.ucla.edu

Daryl Cooper

Department of Mathematics

University of California

Santa Barbara, CA 93106-3080 cooper@math.ucsb.edu

Jiang-Hua $\mathrm{Lu}$

Department of Mathematics

Pokfulam Rd., Hong Kong jhlu@maths.hku.hk
The University of Hong Kong

Don Blasius

Department of Mathematics University of California

Los Angeles, CA 90095-1555

blasius@math.ucla.edu

Robert Finn

Department of Mathematics Stanford University

Stanford, CA 94305-2125

finn@math.stanford.edu

Sorin Popa

Department of Mathematics

University of California

Los Angeles, CA 90095-1555 popa@math.ucla.edu

Paul Yang

Department of Mathematics Princeton University

Princeton NJ 08544-1000

yang@math.princeton.edu

\section{PRODUCTION}

Silvio Levy, Scientific Editor, production@msp.org

\section{SUPPORTING INSTITUTIONS}

ACADEMIA SINICA, TAIPEI

CALIFORNIA INST. OF TECHNOLOGY

INST. DE MATEMÁTICA PURA E APLICADA

KEIO UNIVERSITY

MATH. SCIENCES RESEARCH INSTITUTE

NEW MEXICO STATE UNIV.

OREGON STATE UNIV.

\author{
STANFORD UNIVERSITY \\ UNIV. OF BRITISH COLUMBIA \\ UNIV. OF CALIFORNIA, BERKELEY \\ UNIV. OF CALIFORNIA, DAVIS \\ UNIV. OF CALIFORNIA, LOS ANGELES \\ UNIV. OF CALIFORNIA, RIVERSIDE \\ UNIV. OF CALIFORNIA, SAN DIEGO \\ UNIV. OF CALIF., SANTA BARBARA
}

\author{
Vyjayanthi Chari \\ Department of Mathematics \\ University of California \\ Riverside, CA 92521-0135 \\ chari@math.ucr.edu \\ Kefeng Liu \\ Department of Mathematics \\ University of California \\ Los Angeles, CA 90095-1555 \\ liu@math.ucla.edu \\ Jie Qing \\ Department of Mathematics \\ University of California \\ Santa Cruz, CA 95064 \\ qing@cats.ucsc.edu
}

These supporting institutions contribute to the cost of publication of this Journal, but they are not owners or publishers and have no responsibility for its contents or policies.

See inside back cover or msp.org/pjm for submission instructions.

The subscription price for 2013 is US \$400/year for the electronic version, and \$485/year for print and electronic.

Subscriptions, requests for back issues and changes of subscribers address should be sent to Pacific Journal of Mathematics, P.O. Box 4163, Berkeley, CA 94704-0163, U.S.A. The Pacific Journal of Mathematics is indexed by Mathematical Reviews, Zentralblatt MATH, PASCAL CNRS Index, Referativnyi Zhurnal, Current Mathematical Publications and the Science Citation Index.

The Pacific Journal of Mathematics (ISSN 0030-8730) at the University of California, c/o Department of Mathematics, 798 Evans Hall \#3840, Berkeley, CA 94720-3840, is published twelve times a year. Periodical rate postage paid at Berkeley, CA 94704, and additional mailing offices. POSTMASTER: send address changes to Pacific Journal of Mathematics, P.O. Box 4163, Berkeley, CA 94704-0163.

PJM peer review and production are managed by EditFLOW ${ }^{\circledR}$ from Mathematical Sciences Publishers.

\section{PUBLISHED BY}

mathematical sciences publishers

nonprofit scientific publishing

http://msp.org/

(C) 2013 Mathematical Sciences Publishers 


\section{PACIFIC JOURNAL OF MATHEMATICS}

Volume $265 \quad$ No. $2 \quad$ October 2013

Singularity removability at branch points for Willmore surfaces

YANN BERNARD and TRISTAN RIVIÈRE

On Bach flat warped product Einstein manifolds

QIANG CHEN and CHENXU HE

On plane sextics with double singular points

Alex DeGTyareV

A computational approach to the Kostant-Sekiguchi correspondence

HEIKo DiETRICH and WILLEM A. DE GRAAF

Landau-Toeplitz theorems for slice regular functions over quaternions

GRAZIANO GENTILI and GiUlia SARFATTI

On surgery curves for genus-one slice knots

PATRICK M. GILMER and CHARLES LIVINGSTON

Characterizing abelian varieties by the reduction of the Mordell-Weil group

Chris Hall and Antonella Perucca

Variation of complex structures and the stability of Kähler-Ricci

Solitons

StUART J. HALl and ThOMAS MURPHY

On crossed homomorphisms of the volume preserving diffeomorphism groups

RYOJI KASAGAWA

Regularity at the boundary and tangential regularity of solutions of the Cauchy-Riemann system

TRAN VU KHANH and GIUSEPPE ZAMPIERI

On the Steinberg character of a semisimple $p$-adic group 